\title{
A Message-Scheduling Scheme for Energy Conservation in Multimedia Wireless Systems
}

\author{
Xiaojun Ruan, Associate Member, IEEE, Shu Yin, Student Member, IEEE, Adam Manzanares, Member, IEEE, \\ Mohammed Alghamdi, and Xiao Qin, Senior Member, IEEE
}

\begin{abstract}
Reducing power consumption of wireless networks has become a major goal in designing modern multimedia wireless systems. In an effort to reduce power consumption, this paper addresses the issue of scheduling real-time messages in multimedia wireless networks subject to both timing and power constraints. A power-consumption model is introduced to calculate powerconsumption rates in accordance with message-transmission rates. Next, a new message-scheduling scheme called Power-aware Real-time Message (PARM) is developed to generate messagetransmission schedules that minimize power consumption of multimedia wireless-network interfaces and the probability of missing deadlines for real-time messages. With a power-aware scheduling policy in place, the proposed PARM scheme is very energyefficient. Experimental results based on a wide variety of synthetic workloads and eight real-world applications show that PARM significantly reduces energy dissipation while maintaining low missed rates. PARM reduces power consumption of data transmissions by up to $99.4 \%$ (with an average of $86.7 \%$ ) for synthetic network traffic and saves energy by up to $60.0 \%$ (with an average of $34.1 \%$ ) in the eight real-world applications.
\end{abstract}

Index Terms-Energy conservation, multimedia wireless systems, power-aware scheduling, real-time messages.

\section{INTRODUCTION}

$\mathbf{F}$ UTURE-GENERATION wireless networks seamlessly provide users with multimedia and data services. There have been increasing efforts to develop real-time applications in wireless networks. Advanced interactive multimedia applications are real-time in nature; typical examples of multimedia applications in wireless networks include video telephony, video games, and television broadcasting. [37] Real-time applications in wireless networks depend not only on the logical results of computation but also on the time at which the results are produced [18], [29], [30], [32].

Manuscript received January 4, 2008; revised September 15, 2008 and July 2, 2009; accepted October 4, 2009. Date of publication October 21, 2010; date of current version January 19, 2011. This work was supported in part by the National Science Foundation under Grants CCF-0845257 (CAREER), CNS0757778 (CSR), CCF-0742187 (CPA), CNS-0917137 (CSR), CNS-0831502 (CyberTrust), CNS-0855251 (CRI), OCI-0753305 (CI-TEAM), DUE-0837341 (CCLI), and DUE-0830831 (SFS) and in part by Auburn University under a startup Grant and a gift (2005-04-070) from the Intel Corporation. This paper was recommended by Associate Editor B. Veeravalli.

X.-J. Ruan, S. Yin, and X. Qin are with the Department of Computer Science and Software Engineering, Auburn University, Auburn, AL 36849 USA (e-mail: xzr0001@auburn.edu; szy0004@auburn.edu; xqin@ auburn.edu).

A. Manzanares is with the Los Alamos National Laboratory, Los Alamos, NM 87545 USA (e-mail: acm0008@ auburn.edu).

M. Alghamdi is with the Department of Computer Science, Al-Baha University, Al-Baha 65431, Saudi Arabia (e-mail: alghamdi@nmt.edu).

Color versions of one or more of the figures in this paper are available online at http://ieeexplore.iee. org.

Digital Object Identifier 10.1109/TSMCA.2010.2069095
In addition to handling multimedia traffic like speech and video, challenges in improving wireless-network capability include energy efficiency and different classes of data traffic. The reason is that a large number of wireless devices are multimedia oriented, and most wireless devices have constraints on energy consumption, size, and bandwidth [2], [12], [26]. Recent studies show that battery-performance improvement rate in terms of energy per unit size or weight is fairly slow, and energy consumption needed for communication will severely limit the functionality of wireless networks. Emerging multimedia applications with higher power needs make battery performance even worse. Therefore, reducing power consumption of realtime traffic is an efficient approach to achieve long battery life for wireless networks supporting multimedia applications.

In recent years, energy conservation of processor for realtime systems has been extensively studied. However, energy conservation in wireless networks using scheduling remains an open problem. Such energy-conservation technologies become critical because admission control and scheduling approaches are expected to significantly reduce energy consumption of real-time communication in wireless networks.

Scheduling algorithms play a key role in obtaining high performance in real-time wireless data networks [6], [7], [19]. In addition to high-performance, energy-aware messagescheduling algorithms need to be developed to further improve energy efficiency of wireless networks. This paper proposes an energy-aware scheduling algorithm called Power-aware Realtime Message (PARM) for real-time messages in wireless networks in the context of future-generation multimedia applications. It should be noted that the terms message and packet are used interchangeably throughout this paper. The novelty of the approach lies in the fact that the message-scheduling algorithm aims at meeting timing constraints and conserving power consumption for real-time wireless networks. To quantitatively evaluate the message-scheduling algorithm, a simulation platform is built to compare the approach against four baseline algorithms that are unaware of energy efficiency. Experimental results based on a wide variety of synthetic workloads and eight real-world applications show that PARM significantly reduces energy dissipation while maintaining low missed rates.

The rest of this paper is organized as follows. Related work is discussed in Section II. Section III describes the mathematical models. The new PARM algorithm is proposed in Section IV. An analytical model is constructed in Section V. Section VI presents the simulation platform and experimental results of PARM scheduling scheme for wireless networks. Finally, Section VII concludes this paper. 


\section{RELATED WORK}

Some research has been done on real-time scheduling for real-time systems [1], [14], [20], [29]. In practice, a real-time scheduling algorithm generally falls into two categories: static (offline) [36] and dynamic (online) [28]. Lipari and Buttazzo proposed a framework for scheduling real-time multitask applications in an open system [18]. Qin and Jiang developed an efficient fault-tolerant scheduling algorithm for real-time tasks with precedence constraints [24], [25]. In previous works, Xie and Qin studied security-aware scheduling for real-time applications in embedded systems [36], clusters [34], [35], grids [33], and storage systems [21], [22]. Although these scheduling algorithms achieve high performance for real-time computing, they are inadequate for real-time wireless networks.

Recently, increasing attention has been paid to energyefficiency for wireless networks because high energy-efficiency has become a baseline requirement. Jones et al. examined the current state of the art in energy efficiency in the context of wireless networks and introduced new technologies that meet the energy-efficiency needs of wireless networks [16]. Stine and Veciana studied various methods to improve energy efficiency of centrally controlled wireless data networks [28]. Huang proposed an adaptive and energy-conserving indexing method for on-demand data broadcasting systems [15]. Wang and Wang proposed a new adaptive call admission control scheme that takes energy conservation in wireless networks into account [32]. However, the aforementioned energy-efficiency techniques are not adequate for real-time applications due to the lack of ability to handle timing constraints.

Some works have been done to incorporate energy efficiency into a variety of real-time applications. Havinga and Smit identified prominent problems of wireless multimedia networking and presented various solutions to improve energy efficiency for multimedia applications [13]. Lipari and Buuazzo proposed an approach to trade off quality of energy efficiency to achieve required real-time performance [18]. Akkaya and Younis proposed an energy-aware QoS routing protocol to find a least cost delay-constrained path for real-time data [2]. The PARM work is fundamentally different from the previous approaches because this paper is focused on the issue of energy-aware message scheduling for real-time wireless networks.

Zhu and Cao conducted excellent research on designing power-aware and QoS-aware service models over wireless networks [38], [39]. After constructing a novel power-conserving service model for streaming applications over wireless networks, they investigated the rate-based bulk-scheduling (RBS) algorithm to determine which flow should be served at which time. The RBS algorithm not only makes it possible for wireless network interfaces to sleep for a long time period to conserve energy but also provides delay guarantee. RBS achieves high power efficiency by buffering data so that the wireless network interface can sleep for a long time period to conserve energy. Different from RBS that noticeably reduces power consumption by powering off the wireless-network interfaces of the receivers, PARM provides significant energy savings by lowering the transmission power of the wireless-network interfaces of senders as long as deadlines of real-time messages are guaranteed. It is worth nothing that the PARM algorithm presented in this paper can be considered complementary to the RBS algorithm in the sense that additional energy savings can be achieved by integrating PARM with RBS.

Prabhakar et al. considered packet transmission schedules that minimize energy in wireless networks while warranting deadline or delay constraints [22] and energy-efficient packet transmission over a wireless link [32]. After realizing that power consumption can be substantially reduced by lowering transmission power, Prabhakar et al. proposed the lazy-packetscheduling approach to reduce the transmission rates of packets without violating deadlines. Khojastepour and Sabharwal investigated delay-constrained package schedulers that guarantee a maximum queuing delay for each packet while minimizing power consumption in network systems. The major distinction between the lazy-packet-scheduling and delay-constrained scheduling schemes and PARM is fourfold. First, their schemes assumed that all packets shared a common deadline $T$, i.e., all transmissions must be completed by time $T$. In contrast, PARM algorithm relaxes the assumption by allowing each packet to have an individual deadline. In doing so, PARM is more flexible because a set of real-time packets with a common deadline is a special case of the general packet-scheduling problem addressed in this paper. Second, Prabhakar et al.'s online scheduling approach was built based on their optimal offline scheduling algorithm. Although PARM is also an online scheduling algorithm, the PARM algorithm is developed from ground up, specifically for dynamically arriving packets. Thus, PARM is independent of any optimal offline algorithm. Third, in order to apply the online algorithms designed by Prabhakar et al., one has to acquire the statistics of arrival processes. Unlike their approach, PARM can be used to schedule real-time packets without knowing packet arrival patterns. Fourth, since the aforementioned scheduling approaches assume that the deadlines of all packets are identical, the schedulers treat packets in the same fashion. PARM, on the other hand, assigns higher priority to packets with earlier deadlines. PARM's strategy can guarantee more packets to be transmitted before the corresponding deadlines.

\section{MOdELS}

\section{A. Message-Scheduling Architecture}

A base station in a wireless network communicates with mobile terminals via downlink and uplink channels. It is assumed in this paper that an uplink channel can be granted through a downlink or randomly accessed by mobile terminals, and download channels are accessed using the time division multiple access (TDMA) method. In TDMA, different users are allowed to share the same frequency channel by dividing the signal into different time slots. Similar assumptions are used in many existing works [39]. The PARM mechanism in the system architecture shown in Fig. 1 is mainly composed of an energyconsumption controller (ECC), an admission controller (AC), and an earliest deadline first (EDF) scheduler (where an EDF scheduling algorithm is applied). Although the EDF scheduling strategy is simplistic, it is still one of the most widely used 


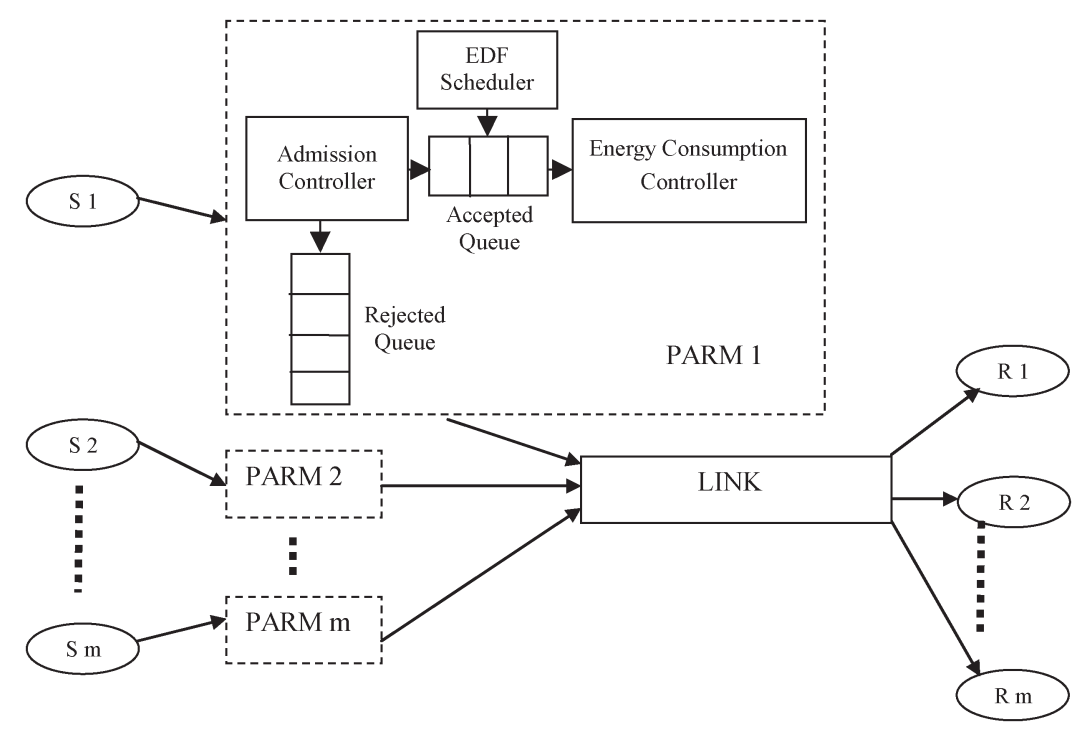

Fig. 1. Architecture of the PARM Strategy.

scheduling algorithms [3], [4], [8]. The PARM mechanism is deployed in the wireless network interface of each sender, which delivers incoming messages to receivers through a wireless link. All the messages are independently submitted from $m$ mobile terminals, and their arrival times abide by Poisson distribution. The function of the $\mathrm{AC}$ is to determine if an incoming message can be accepted or not, whereas the ECC is intended to minimize the energy consumption of current messages residing in an accepted queue. The EDF scheduler transmits the messages in accordance with their deadlines in a way that messages with the earliest deadlines are given the highest priority. If a real-time message's deadline cannot be guaranteed, the message will be dropped in the rejected queue.

\section{B. Modeling Real-Time Messages}

The PARM real-time message model assumes that all messages have soft deadlines, and all messages are independent of one another. In a PARM system model, there exists a PARM mechanism that handles real-time messages from each mobile terminal. Each message has a SenderID that indicates the identity of the message sender. Likewise, each message has a ReceiverID that hints where the message should be delivered. Messages arrive at a PARM mechanism according to a Poisson process. Message $m_{i}$ is represented as a tuple $\left(a_{i}, f_{i}, \operatorname{sid}_{i}, \operatorname{rid}_{i}\right.$, $s_{i}, D_{i}$ ), where $a_{i}$ and $f_{i}$ are the arrival time and finish time of message $i . \operatorname{sid}_{i}$ and $\operatorname{rid}_{i}$ represent the identity of the message sender and the identity of message receiver, respectively. $s_{i}$ is the data size measured in bits. $D_{i}$ is the relative deadline of message $m_{i}$. The absolute deadline $d_{i}$ of $m_{i}$ is determined by the arrival time and relative deadline of $m_{i}$, i.e., $d_{i}=a_{i}+D_{i}$. Note that, given a message $m_{i}$, all elements in the tuple of $m_{i}$ are known a priori. The transmission time of message $m_{i}$ can be computed by where $b_{i}$ is the transmission rate of message $m_{i}$. Let $M=$ $\left\{m_{1}, m_{2}, \ldots, m_{n}\right\}$ be a set of real-time messages submitted to a PARM mechanism.

The PARM power-aware scheduler presented in the following section makes use of a function to measure the energy consumption of each admitted message. Given a message $m_{i}$, its energy consumption $i$ can be quantitatively computed by

$$
\Psi_{i}=s_{i} * \theta_{i}
$$

where $\theta_{i}$ is the energy-consumption rate measured in energy consumption of each transmitted bit and $s_{i}$ is the data size of $m_{i}$. The value of $i$ depends on the transmission rate of the current message $m_{i}$. Hence, the energy consumption of one message can be calculated using (2). The energy-consumption rate is a function of the transmission rate $b_{i}$, i.e., $\theta_{i}=\Gamma\left(b_{i}\right)$. The function $\Gamma$ [see (3)] is obtained by taking the points from a previous study [32] and fitting a polynomial curve to the experimental points

$$
\begin{aligned}
\Gamma\left(b_{i}\right)=-65.94- & 0.012 b_{i}+7.656 \times 10^{-5} b_{i}^{2} \\
- & 1.643 \times 10^{-7} b_{i}^{3}+1.451 \times 10^{-10} b_{i}^{4} .
\end{aligned}
$$

The function $\Gamma$ used to compute the energy-consumption rate is shown in Fig. 2 (see Section III-C). Note that the energy-consumption rates used in this paper are expressed in terms of decibel (dB) values. Let $X_{i}$ be all possible schedules for message $m_{i}$ and $x_{i} \in X_{i}$ be a scheduling decision of $m_{i} . x_{i}$ is a feasible schedule if deadline $d_{i}$ can be met, i.e., $f_{i} \leq d_{i}$. Given a real-time message $m_{i}$, the economical energy consumption of the wireless network interface with respect to $m_{i}$ is minimized by the ECC (see Fig. 1) under the timing constraint of $m_{i}$. In other words, the economical energy consumption $\Psi_{i}\left(X_{i}\right)$ is the minimal energy consumption among all energy consumption of the possible schedulers. Thus

$$
\Psi_{i}\left(X_{i}\right)=\min _{x_{i} \in X_{i}}\left\{\Psi_{i}\left(x_{i}\right)\right\}
$$

$$
t_{i}=s_{i} / b_{i}
$$






Fig. 2. Energy-consumption model.

An energy-aware scheduler strives to minimize a wirelessnetwork interface's energy consumption. Let $X$ be all possible schedules for the entire message set $M$. Thus, the total energyconsumption function needs to be minimized subject to timing constraints

$$
\Psi(X)=\min _{x \in X}\left\{\sum_{i=1}^{n} \Phi\left(x_{i}\right) \cdot \Psi_{i}\left(x_{i}\right)\right\}
$$

where $n$ is the number of submitted messages and $\Phi$ is a step function, i.e., $\Phi\left(x_{i}\right)$ is set to one if $m_{i}$ is admitted and is set to zero, otherwise. Equation (5) signifies that the energy consumption of a wireless-network interface can be computed as the sum of the power consumption of all messages admitted to the network interface.

\section{Energy-Consumption Model}

Equation (3) and Fig. 2 show the relationship between the power-consumption rate and the message-transmission rate. The vertical axis is the power-consumption rate, whereas the horizontal axis is the message-transmission rate measured in kilobits per second. The energy-consumption model is built based on the empirical results reported in [32]. The result in [32] is extended by using curve fitting, so it is possible to quantitatively obtain the corresponding power consumption when the message-transmission rate varies in the range between 125 and $1000 \mathrm{~kb} / \mathrm{s}$. This paper pays attention to nonexceptional cases where energy consumption can be efficiently conserved by reducing transmission rate. Like several existing models reported in the literature [11], [27], the PARM model does not account for energy consumption for retransmissions. Thus, the PARM energy-consumption model is suitable for a wireless link with a single transmission attempt across the link. It is worth nothing that the energy-consumption model used in this paper is for illustration purpose only; therefore, the model may be replaced by any model that not only considers retransmissions but also characterizes the relation between energy consumption and data rate in a mathematical form depending on coding schemes, modulation levels, multiple access methods, and channel types. The PARM scheduling algorithm leverages the energy-consumption model to calculate rates of power con- sumption according to message-transmission rates determined by the ECC.

\section{PARM SCHEDUling Algorithm}

The PARM scheduling algorithm integrates the EDF scheduling algorithm into the energy-consumption heuristic scheme described in Section III.

The basic strategy of PARM is to set the transmission rate of the current message as low as possible without causing any other messages in the admitted queue to miss their deadlines. According to Fig. 2, although using a lower transmission rate costs more time for a message, the energy consumption will be lower because the power rate increases exponentially with the transmission rate. Therefore, the key point here is trying to reduce the transmission rate of each message and also making sure that the missed rate of all messages is not significantly increased. In many cases, the missed rate generated by PARM is even lower when compared with many other scheduling strategies.

To facilitate the presentation of the proposed algorithm, the following properties are introduced. The schedule of a message is feasible if the message can be delivered before its deadline. In other words, a message has a feasible schedule if it can be finished at the highest transmission rate. Formally, this fact can be expressed by the following property.

Property 1: If message $m_{i}$ has a feasible schedule, the following inequality must be satisfied: $\omega_{i}+t_{i}^{\min } \leq d_{i}$, under the condition that $\forall m_{k} \in A Q, d k>d_{i}: w_{k}+t_{k}^{\min } \leq d_{k}$, where $A Q$ is the accepted queue, $\omega$ is the earliest transmission start time of message $m_{i}$, and $t_{i}^{\min }$ is the minimal transmission time of message $m_{i}$ when its transmission rate is set to the highest value. The condition enforced in Property 1 indicates that the transmission of $m_{i}$ results in no violation of any deadlines of messages that have been admitted to the PARM mechanism.

The PARM algorithm is nontrivial because PARM must dynamically estimate the amount of time it will take to transmit a message given what PARM currently knows about the state of the wireless network. One way to estimate the transmission time of message $m_{i}$ is based on the fact that message $m_{i}$ cannot start its transmission unless all messages with earlier deadlines have been delivered. Thus, the transmission time of the message is equal to the summation of times spent on transmitting message $m_{i}$ and all the messages whose deadlines are earlier than that of $m_{i}$. Hence, the earliest transmission start time $\omega_{i}$ can be accurately computed by

$$
\omega_{i}=r+\sum_{m_{k} \in M, d_{k} \leq d_{i}} \frac{s_{k}}{b_{k}}
$$

where $r$ represents the current time and $s_{k} / b_{k}$ is the transmission time of message $m_{i}$ whose deadline is earlier than that of $m_{i}$. Thus, the earliest transmission start time of $m_{i}$ is the sum of the remaining transmission time of a message being transmitted and the transmission time of the messages with earlier deadlines.

The PARM algorithm is described as follows. The goal of the PARM algorithm is to achieve low energy consumption of a wireless link in a multimedia network system while 


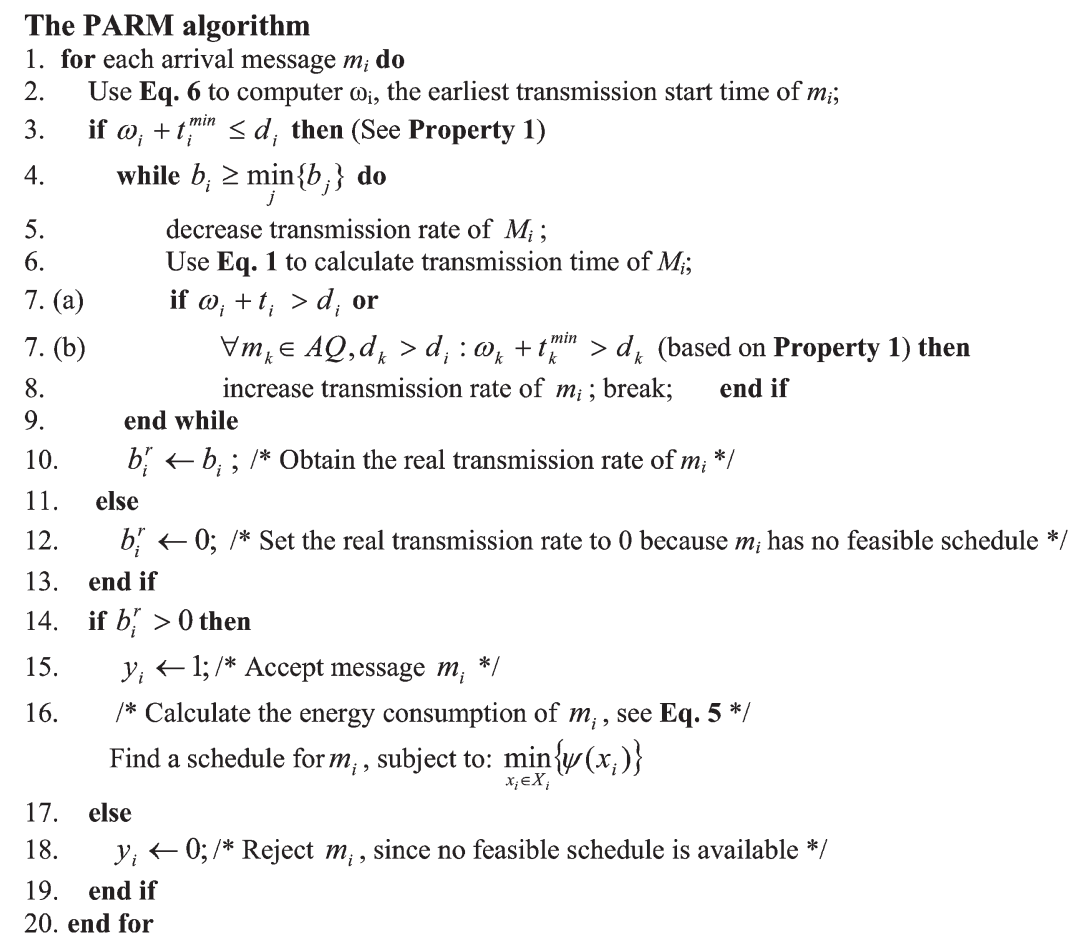

guaranteeing timing constraints of real-time messages being delivered through the link. More specifically, PARM aims at reducing wireless transmission rate to minimize energy dissipation [measured by (2)] caused by each admitted real-time message (see Step 16) while maintaining a reasonably high guarantee ratio, which is measured by the fraction of the total arrived messages that are found to be schedulable (see Step 3). A salient feature of PARM is that the real-time requirement of a message will be guaranteed if it is admitted to the wireless link by PARM, indicating that PARM conserves energy in a wireless link without significantly degrading real-time performance.

The time complexity of scheduling message $m_{i}$ by PARM is $O(k n)$, where $n$ is the number of transmission rates and $k$ is the worst case number of messages whose deadlines are earlier than that of $m_{i}$. The time complexity is $O(k n)$ because the time complexity of computing $\omega_{i}$ is $O(k)$ (see Step 2), and it takes $O(n)$ time to minimize transmission rate (see Steps 4-9). The time complexity is very low since $n$ in almost all cases is a small value. It is necessary to consider all the messages whose deadlines are greater than $m_{i}$ 's deadline because of the following reasons. When the algorithm is checking the deadline constraints for $m_{i}$, PARM must figure out whether the deadlines of those accepted messages whose deadlines are greater than $m_{i}$ are violated. Step 7(b) needs to recalculate the earliest transmission times and the transmission rates of those accepted messages whose deadlines are earlier than $m_{i}$.

Before optimizing the energy consumption of message $m_{i}$, PARM attempts to meet the real-time requirement of $m_{i}$ by calculating the earliest start time [using (6)] in Step 2. Then, PARM performs a feasibility check, figuring out if $m_{i}$ can be successfully delivered before its deadline (see Step 3). When the deadline is missed, $m_{i}$ is rejected by the PARM algorithm (see Step 18).
The energy consumption of message $m_{i}$ is optimized in Step 5, which decreases the transmission rate of $m_{i}$ while satisfying the following two conditions. First, decreasing the transmission rate of $m_{i}$ will not miss the deadline of $m_{i}$. Second, the increment of the transmission time of $m_{i}$ due to the reduced transmission rate must not result in missing deadlines of any previously admitted messages. These two conditions are respectively satisfied by Steps 7(a) and 7(b). Once Step 10 has dynamically decided the transmission rate of message $m_{i}$, Step 16 can calculate the energy consumption for the chosen schedule of $m_{i}$ (see Step 16).

\section{PERFormanCE ANALYsis}

In this section, an analytical model is constructed to provide probabilistic performance guarantees for real-time messages. First, PARM calculates the expected power consumption used by a node per real-time message with security requirements. Second, PARM derives the probability that a real-time message is transmitted before its absolute deadline.

\section{A. Analysis of Power Consumption}

To analyze the performance of PARM in power consumption, the expected energy $E(\Psi)$ (measured in joules) is derived by a node per real-time message delivered. The transmission rate of a real-time message is a random variable denoted by $B$. Let us first compute the probability $\operatorname{Pr}(B=x)$ that the transmission rate is chosen to be $x$. Recall that the transmission rate of a real-time message is determined based on the data size and deadline of the message and transmission times of messages with earlier deadlines to be delivered. The data size, deadline, the earliest transmission start time are modeled as random variables denoted by $S, D$, and $\Omega$. Note that $S, D$, and $\Omega$ 
are distributed according to three probability density functions, which are known a priori. Let the data size, deadline, and the earliest transmission start time of a newly arrived message be $i, j$, and $k$. The transmission rate of the message is determined subject to the following two timing constraints:

$$
k+\frac{i}{x} \leq j, \quad \frac{i}{x} \leq \min _{d_{l}>j}\left(\mu_{l}\right)
$$

where $\mu_{l}$ is the slack time of a message whose deadline is larger than $j$. The slack time is defined as the time period between the start time of the current message and the latest allowable start time of the other message. In other words, a message has to start before $k+\mu_{l}$; otherwise, the deadline will not be satisfied even if the message is transmitted under the highest transmission rate. The slack time in PARM is the utilizable time for each message, which means that if the current message transmission can be completed in this period of time, the start time of the following message will not be affected. The first condition in (7) guarantees that the newly arrived message can be transmitted before its deadline $j$, and the second condition ensures that the messages whose deadlines are later than $j$ can be delivered before their respective deadlines. Based on the earlier two timing constraints, it is possible to calculate the probability $\operatorname{Pr}=(B=x \mid S=i, D=j, \Omega=k)$ as follows:

$$
\begin{aligned}
& \operatorname{Pr}(B=x \mid S=i, D=j, \Omega=k) \\
& =\operatorname{Pr}\left(k+\frac{i}{x} \leq j, \frac{i}{x} \leq \min _{d_{l}>j}\left(\mu_{l}\right) \mid S=i, D=j, \Omega=k\right) .
\end{aligned}
$$

Thus, the probability $\operatorname{Pr}(B=x)$ that the transmission rate is chosen to be $x$ is computed by the following:

$$
\operatorname{Pr}(B=x)=\sum_{i, j, k}[\operatorname{Pr}(B=x \mid S=i, D=j, \Omega=k)] .
$$

The probability $\operatorname{Pr}(B=x)$ in ID: (9) is computed by (10) and (11), shown at the bottom of the page. Recall that the energy-consumption rate is a function of the transmission rate, i.e., $\theta=\Gamma(b)$. Hence, given an energy-consumption rate, the corresponding transmission rate can be expressed as $b=\Gamma_{-1}(\theta)$. The energy-consumption rate is treated as a random variable denoted by $\Theta$. The probability that the energy-consumption rate is equal to $\theta$ can be computed by (9), where the expression on the right-hand side of the equation is obtained by ( 8 )

$$
\operatorname{Pr}(\Theta=\theta)=\operatorname{Pr}\left(B=\Gamma^{-1}(\theta)\right) .
$$

Now, the expected energy $E(\Psi)$ per real-time message delivered is derived as

$$
\begin{aligned}
E(\Psi) & =\sum_{\theta}[\theta \cdot \operatorname{Pr}(\Theta=\theta) \cdot E(S)] \\
& =\sum_{\theta}\left\{\theta \cdot \operatorname{Pr}(\Theta=\theta) \cdot \sum_{i}[i \cdot \operatorname{Pr}(S=i)]\right\} \\
& =\sum_{\theta=\Theta_{\min }}^{\Theta_{\max }}\left\{\theta \cdot \operatorname{Pr}(\Theta=\theta) \cdot \sum_{i=S_{\min }}^{S_{\max }}[i \cdot \operatorname{Pr}(S=i)]\right\} \\
& =\sum_{\theta=\Theta_{\min }}^{\Theta_{\max }}\left\{\theta \cdot \operatorname{Pr}(\Theta=\theta) \cdot \sum_{i=S_{\min }}^{S_{\max }}\left[i \cdot h_{i}\right]\right\}
\end{aligned}
$$

$$
\begin{aligned}
\operatorname{Pr}(B=x) & =\sum_{i, j, k}[\operatorname{Pr}(B=x \mid S=i, D=j, \Omega=k)] \\
& =\sum_{i, j, k}\left[\operatorname{Pr}\left(k+\frac{i}{x} \leq j, \frac{i}{x} \leq \min _{d_{l}>J}\left(\mu_{l}\right) \mid S=i, D=j, \Omega=k\right)\right] \\
& =\sum_{i=S_{\min }}^{S_{\max }}\left\{\operatorname{Pr}(S=i) \cdot \sum_{j, k}\left[\operatorname{Pr}\left(k+\frac{i}{x} \leq j, \frac{i}{x} \leq \min _{d_{l}>j}\left(\mu_{l}\right) \mid D=j, \Omega=k\right)\right]\right\} \\
& =\sum_{i=S_{\min }}^{S_{\max }}\left\{\operatorname{Pr}(S=i) \cdot \sum_{j=D_{\min }}^{D_{\max }}\left[\operatorname{Pr}(D=j) \sum_{k}\left(\operatorname{Pr}\left(k+\frac{i}{x} \leq j, \frac{i}{x} \leq \min _{d_{l}>j}\left(\mu_{l}\right) \mid \Omega=k\right)\right)\right]\right\} \\
& =\sum_{i=S_{\min }}^{S_{\max }}\left\{\operatorname{Pr}(S=i) \cdot \sum_{j=D_{\min }}^{D_{\max }}\left[\operatorname{Pr}(D=j) \cdot \sum_{k=0}^{\infty}\left(\operatorname{Pr}(\Omega=k) \cdot \operatorname{Pr}\left(k+\frac{i}{x} \leq j, \frac{i}{x} \leq \min _{d_{l}>j}\left(\mu_{l}\right)\right)\right)\right]\right\} \\
& =\sum_{i=S_{\min }}^{S_{\max }}\left\{\operatorname{Pr}(S=i) \cdot \sum_{j=D_{\min }}^{D_{\max }}\left[\operatorname{Pr}(D=j) \cdot \sum_{k=0}^{\infty}\left(\operatorname{Pr}(\Omega=k) \cdot \operatorname{Pr}\left(k+\frac{i}{x} \leq j\right) \cdot \operatorname{Pr}\left(\frac{i}{x} \leq \min _{d_{l}>j}\left(\mu_{l}\right)\right)\right)\right]\right\} \\
\operatorname{Pr}(B=x) & =\sum_{i=S_{\min }}^{S_{\max }}\left\{\sigma_{i} \cdot \sum_{j=D_{\min }}^{D_{\max }}\left[\delta_{j} \cdot \sum_{k=0}^{\infty}\left(\xi_{k} \cdot \operatorname{Pr}\left(k+\frac{i}{x} \leq j\right) \cdot \operatorname{Pr}\left(\frac{i}{x} \leq \min _{d_{l}>j}\left(\mu_{l}\right)\right)\right)\right]\right\}
\end{aligned}
$$


where $\Theta_{\min }$ and $\Theta_{\max }$ are the minimum and maximum energyconsumption rates. Let $p_{\theta}$ denote the probability $\operatorname{Pr}(\Theta=\theta)$ that the energy-consumption rate is equal to $\theta$. The probability $h_{i}$ in (13) is computed by (14) as follows:

$$
\begin{aligned}
h_{i}= & \operatorname{Pr}\left(\frac{s_{i}}{b_{i}}=1 \mid \omega_{i} \geq d_{i}\right) \\
& \vdots \\
& +\operatorname{Pr}\left(\frac{s_{i}}{b_{i}}=k \mid \omega_{i} \geq d_{i}+1-k\right) \\
& \vdots \\
& +\operatorname{Pr}\left(\frac{s_{i}}{b_{i}}=d_{i}+1 \mid \omega_{i} \geq 0\right) \\
= & u_{1} \sum_{y=d_{i}}^{\infty} v_{y}+u_{2} \sum_{y=d_{i}-1}^{\infty} v_{y}+\cdots+u_{d_{i}} \sum_{y=1}^{\infty} v_{y}+u_{d_{i}+1} \sum_{y=0}^{\infty} v_{y} \\
= & \sum_{x=1}^{d_{i}+1}\left(u_{x} \sum_{y=d_{i}+1-x}^{\infty} v_{y}\right)
\end{aligned}
$$

where the second line in the previous expression is the conditional probability that the transmission time of $m_{i}$ is $k$, given that it requires at least $d_{i}+1-k$ time unit to deliver the messages with earlier deadlines. $\sum_{j=1}^{i-1}\left(s_{j} / b_{j}\right)$ is a sum of $i-1$ random variables. Since the density function of the sum of random variables is equal to the convolution of the density functions of the individual terms of the sum, the probability $v_{y}=\operatorname{Pr}\left(r+\sum_{j=1}^{i-1}\left(s_{j} / b_{j}\right)=y\right)$ used in (14) is given by

$$
\begin{aligned}
v_{y}= & \operatorname{Pr}\left(\omega_{i}=y\right)=\operatorname{Pr}\left(r+\sum_{j=1}^{i-1} \frac{s_{j}}{b_{j}}=y\right) \\
= & \operatorname{Pr}\left(\sum_{j=1}^{i-1} \frac{s_{j}}{b_{j}}=y-r\right) \\
= & \sum_{k_{1}} \operatorname{Pr}\left(\frac{s_{1}}{b_{1}}=k_{1}\right) \cdot \sum_{k_{2}} \operatorname{Pr}\left(\frac{s_{2}}{b_{2}}=k_{2}\right) \\
& \ldots \sum_{k_{i-2}} \operatorname{Pr}\left(\frac{s_{i-2}}{b_{i-2}}=k_{i-2}\right) \operatorname{Pr}\left(\frac{s_{i-1}}{b_{i-1}}=y-r-\sum_{j=1}^{i-2} k_{j}\right) \\
= & \sum_{k_{1} \cdots k_{i-2}} \prod_{l=1}^{i-2} \operatorname{Pr}\left(\frac{s_{l}}{b_{l}}=k_{l}\right) \operatorname{Pr}\left(\left(\frac{s_{i-1}}{b_{i-1}}=y-r-\sum_{j=1}^{i-2} k_{j}\right)\right) .
\end{aligned}
$$

The probability $u_{x}=\operatorname{Pr}\left(\left(s_{i} / b_{i}\right)=x\right)$ in (13) can be obtained by

$$
\begin{aligned}
u_{x} & =\operatorname{Pr}\left(\frac{s_{i}}{b_{i}}=x\right) \\
& =\sum_{i}\left[\operatorname{Pr}(S=i) \cdot \operatorname{Pr}\left(B=\frac{i}{x}\right)\right] \\
& =\sum_{i=S_{\min }}^{S_{\max }}\left[\sigma_{i} \cdot \operatorname{Pr}\left(B=\frac{i}{x}\right)\right]
\end{aligned}
$$

where $\sigma_{i}=\operatorname{Pr}(S=i)$ and $\operatorname{Pr}(B=(i / x))$ is given by (10).
After probabilities $h_{i}, v_{y}$, and $u_{x}$ are derived from (14)-(16), the expected energy per message expressed in (13) can be calculated as

$$
E(\Psi)=\sum_{\theta=\Theta_{\min }}^{\Theta_{\max }}\left[\theta \cdot p_{\theta} \cdot \sum_{i=S_{\min }}^{S_{\max }}\left(i \cdot \sigma_{i}\right)\right] .
$$

\section{B. Analysis of Guarantee Ratio}

PARM then calculates the probability that a newly arrived message $m_{i}$ can be finished before its deadline $d_{i}$, i.e., $\operatorname{Pr}\left(f_{i} \leq\right.$ $\left.d_{i}\right)$. Before proceeding to the derivation of $\operatorname{Pr}\left(f_{i} \leq d_{i}\right)$, PARM has to consider the probability that $m_{i}$ 's deadline is missed. There are two cases where $m_{i}$ 's deadline cannot be guaranteed.

C1) $m_{i}$ 's deadline cannot be met even if $m_{i}$ is transmitted at the highest transmission rate.

C2) Although $m_{i}$ 's deadline can be satisfied, at least one message $m_{j}$ whose deadline is later than $d_{i}$ fails to be delivered before its deadline $d_{j}$.

Let $h_{i}$ and $g_{i}$ denote the probabilities that $\mathbf{C 1}$ and $\mathbf{C 2}$ occur, respectively. With $h_{i}$ and $g_{i}$, the probability $\operatorname{Pr}\left(f_{i}>d_{i}\right)$ that $m_{i}$ 's deadline is missed can be expressed by

$$
\operatorname{Pr}\left(f_{i}>d_{i}\right)=h_{i}+\left(1-h_{i}\right) \cdot g_{i}
$$

where $g_{i}$ is the probability that at least one message $m_{j}$ whose deadline is later than $d_{i}$ fails to be delivered before deadline $d_{j}$. The probability $g_{i}$ is given by

$$
g_{i}=1-\prod_{k=i+1}^{n}\left(1-\bar{g}_{k}\right)
$$

where $\bar{g}_{k}$ is the probability that the $k$ th message's deadline is missed due to the arrival of $m_{i}$. Let $c_{x}=\operatorname{Pr}\left(\omega_{i}+\left(s_{i} / b_{i}\right)=x\right)$ be the probability that $m_{i}$ 's finish time is equal to $x$ and $\bar{c}_{y}=$ $\operatorname{Pr}\left(\sum_{j=i+1}^{k}\left(s_{j} / b_{j}\right)=y\right)$ be the probability that it takes $y$ time units to transmit the $(i+1)$ th, $(i+2)$ th, and $k$ th messages. Thus, the probability $\bar{g}_{k}$ can be computed using $c_{x}$ and $\bar{c}_{y}$

$$
\begin{aligned}
\bar{g}_{k}= & c_{1} \sum_{y=d_{k}}^{\infty} \bar{c}_{y}+c_{2} \sum_{y=d_{k}-1}^{\infty} \bar{c}_{y}+ \\
= & \sum_{x=1}^{d_{k}+1}\left(c_{x} \cdot \sum_{y=d_{k}-x+1}^{\infty} \bar{c}_{y}\right) \\
& \cdots+c_{x} \sum_{y=d_{k}+1-x}^{\infty} \bar{c}_{y}+\cdots+c_{d_{k}+1} \sum_{y=0}^{\infty} \bar{c}_{y} .
\end{aligned}
$$

Recall that the scheduling policy employed in PARM is the EDF policy, which means that messages with earlier deadlines must be assigned higher priorities and be transmitted first. Suppose that the $n$ waiting messages to be delivered are indexed by their priorities, i.e., $\forall 1 \leq i, j \leq n: i<j \Rightarrow d_{i}<d_{j}$. Let $u_{x}$ 's be the probability that message $m_{i}$ 's transmission time is $x$, i.e., $u_{x}=\operatorname{Pr}\left(\left(s_{i} / b_{i}\right)=x\right)$. Assume that the transmission times of the different messages are statistically independent. Let $v_{y}$ be the probability that the earliest transmission start time $\omega_{i}$ 
TABLE I

CharaCteristics OF SyStem Parameters

\begin{tabular}{|c|c|}
\hline Parameter & Value \\
\hline Networks bandwidth & $125-1000 \mathrm{Kbps}$ \\
Minimal deadline base & 100 Seconds \\
Maximal deadline base & 500 Seconds \\
Min data size & $500 \mathrm{~KB}$ \\
Max data size & $1000 \mathrm{~KB}$ \\
Arrival rate & $0.1-1.0 \mathrm{No} . / \mathrm{Sec}$. \\
Number of messages & 10000 \\
\hline
\end{tabular}

is $y$, i.e., $v_{y}=\operatorname{Pr}\left(\omega_{i}=y\right)=\operatorname{Pr}\left(r+\sum_{j=1}^{i-1}\left(s_{j} / b_{j}\right)=y\right)$ [see (5)]. Note that the probabilities $h_{i}$ and $g_{i}$ in (18) are computed by (14) and (19).

Using (14) along with (19), PARM obtains the probability $\operatorname{Pr}\left(f_{i}>d_{i}\right)$ that $m_{i}$ 's deadline is unable to be met [see (18)]. Therefore, the probability that $m_{i}$ can be transmitted before its deadline $d_{i}$ is given by

$$
\operatorname{Pr}\left(f_{i} \leq d_{i}\right)=1-\operatorname{Pr}\left(f_{i}>d_{i}\right) .
$$

\section{Simulation Results}

\section{A. Performance Metrics and Parameters}

To evaluate the proposed PARM algorithm quantitatively, PARM is compared against the following four baseline algorithms, namely minimum first in-first out (FIFO) (MIN_FIFO), minimum EDF (MIN_EDF), maximum FIFO (MAX_FIFO), and maximum EDF (MAX_EDF). Although the basic ideas of the EDF [3], [4], [8] scheduling strategy and the FIFO [5], [8] scheduling strategy are relatively simplistic, they are widely used today as efficient and effective scheduling strategies. The four baseline scheduling algorithms are briefly described in the following.

1) MIN_FIFO: Admitted messages are transmitted based on the FIFO policy. For each admitted message, the MIN_FIFO algorithm assigns the lowest bandwidth (e.g., $125 \mathrm{~kb} / \mathrm{s}$ ), which leads to the lowest energy consumption.

2) MIN_EDF: Messages admitted by the system are delivered using the EDF policy. Similar to the MIN_FIFO algorithm, MIN_EDF transmits each admitted message using the lowest bandwidth.

3) MAX_FIFO: The FIFO policy is employed to schedule messages, and the highest bandwidth (e.g., $1 \mathrm{Mb} / \mathrm{s}$ ) is assigned to all admitted messages.

4) MAX_EDF: The EDF policy is used to schedule all admitted real-time messages. Regarding transmission-rate assignment, MAX_EDF allocates the highest bandwidth to admitted messages. Table I summarizes the system parameters of the simulated wireless network used in the experiments. Although the data size of messages, deadlines, and arrival rate are synthetically generated, it is still possible to evaluate impacts of these workload parameters on performance by controlling them as fundamental simulation parameters. The performance metrics include the following.

a) Missed rate $(M R)$ : measured as a fraction of total submitted messages that missed their deadlines.

b) Average power consumption.

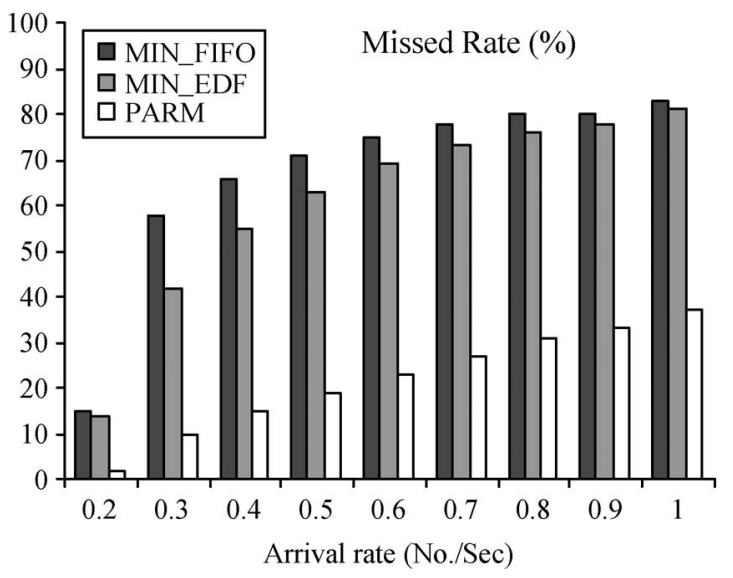

Fig. 3. Missed rate versus arrival rate. Deadline range $=[100,500]$; data size $=[500,1000]$.

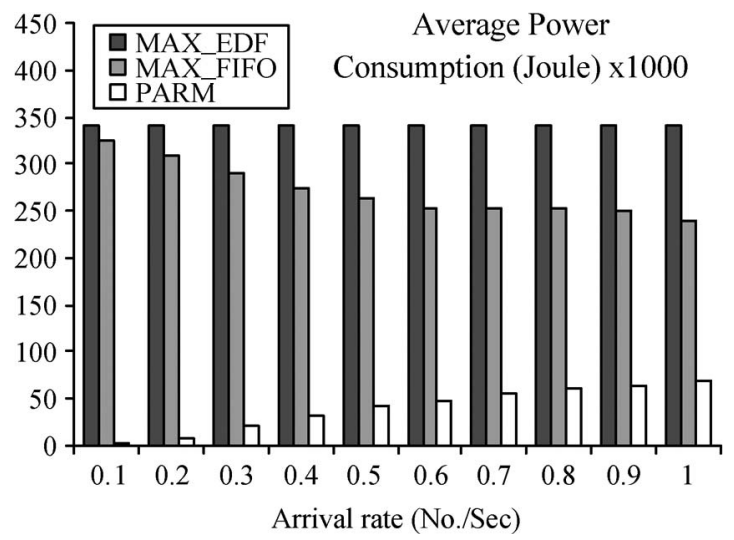

Fig. 4. Average power consumption versus arrival rate. Deadline range $=$ $[100,500]$; data size $=[500,1000]$.

c) Overall system performance: defined by $1-w_{\mathrm{mr}} \mathrm{MR}-w_{\mathrm{pc}} \mathrm{NPC}$, where $w_{\mathrm{mr}}$ and $w_{\mathrm{pc}}$ are two weights and NPC is the normalized power consumption,. In the experiments, $w_{\mathrm{mr}}$ and $w_{\mathrm{pc}}$ are set to 0.5 (see Fig. 7 and Section VI-D).

\section{B. Effect of Arrival Rate}

Figs. 3 and 4 show the simulation results for these five algorithms. The deadlines are randomly generated in the range between 100 and $500 \mathrm{~s}$, and the data size is randomly chosen in the range from 500 to $1000 \mathrm{kB}$. In order to test the PARM algorithm and to prove the correctness of PARM, a synthetic data trace, which can reflect various input parameters for the experiments, is chosen to be used. If all synthetic data traces can produce reasonable results, then PARM should be able to work well in the real world. The missed-rate values of PARM are close to that of MAX_FIFO; the missed-rate values of MAX_EDF are close to $0 \%$. The missed rates of MAX_FIFO and MAX_EDF are omitted from the subsequent figures. Similarly, the power-consumption values of MIN_FIFO and MIN_EDF are almost identical to those of PARM. The power-consumption results of MIN_FIFO and MIN_EDF are not presented in the subsequent sections. 


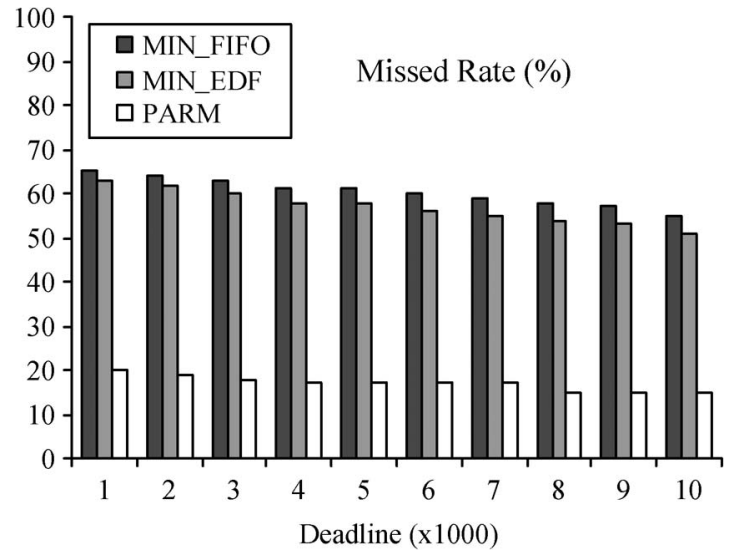

Fig. 5. Missed rate versus deadline. Arrival rate $=0.5$ no./s; data size $=$ $[500,1000]$.

First and foremost, Fig. 3 shows that MIN_EDF performs better than MIN_FIFO, and PARM significantly improves performance in terms of missed rate over both MIN_FIFO and MIN_EDF. This is mainly because MIN_FIFO and MIN_EDF assign the minimal transmission rate to admitted messages resulting in maximal transmission times. Fig. 3 shows that the missed rates of the three algorithms increase with increasing value of arrival rate.

Fig. 4 shows the average power consumption of the MAX_FIFO, MAX_EDF, and PARM when the deadline base is increased from 100 to $500 \mathrm{~s}$. The power consumption of MAX_EDF is almost constant when the arrival rate increases from 0.1 to 1 . The reason is that, MAX_EDF is such an efficient real-time scheduling policy that, very likely, there is no message dropped during the experiments. Hence, the energy consumption of MAX_EDF does not change much in most cases. The energy consumption of MAX_FIFO decreases slightly when the arrival rate is increasing because MAX_FIFO_a nonrealtime scheduling policy-is unable to guarantee many messages' deadlines under high workload conditions. Messages with missing deadlines must be dropped by PARM, making no contribution to the energy consumption. Thus, when the arrival rate goes up, an increasing number of messages with missing deadlines are dropped, thereby leading to reduced power consumption.

Fig. 4 shows that PARM is much more energy-efficient than MAX_FIFO and MAX_EDF. The reason of the performance improvement of PARM is that the PARM scheme noticeably reduces energy consumption by assigning transmission rate for each admitted messages judiciously. Consequently, the PARM algorithm significantly outperforms the other four alternatives in terms of overall performance.

\section{Effect of Deadlines}

Figs. 5 and 6 show the impact of deadlines on the performance of real-time wireless networks. The observation drawn from Fig. 5 is that the missed rates of the algorithms decrease with increasing value of deadlines. This is mainly because loose deadlines enable the AC to accept more real-time messages to be transmitted in the wireless network Fig. 6 shows that the energy consumptions of the three algorithms slightly decrease

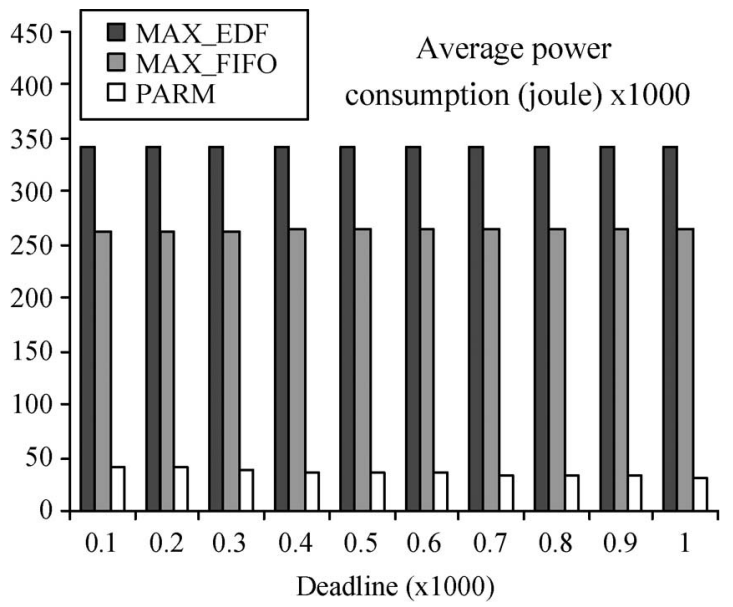

Fig. 6. Average power consumption versus deadline. Arrival Rate $=$ 0.5 no./s; data size $=[500,1000]$.



Fig. 7. Missed rate versus data size. Arrival rate $=0.5$ no./s; deadline range $=[100,500]$.

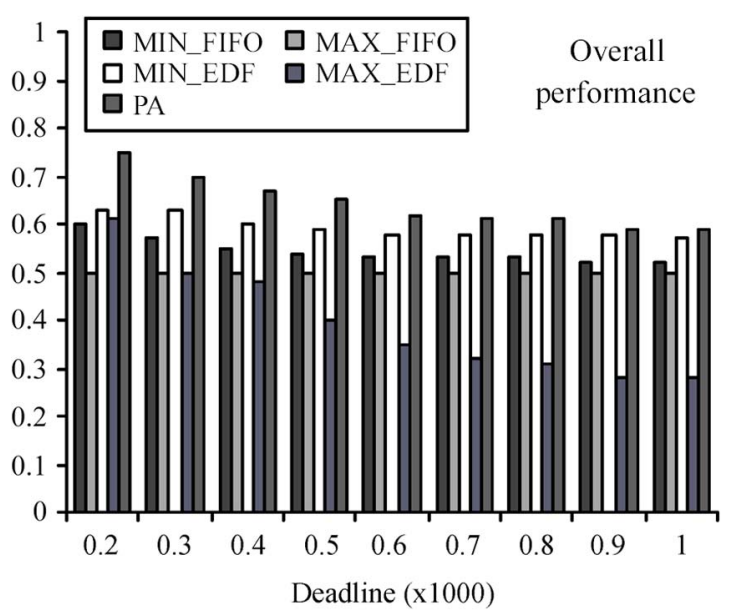

Fig. 8. Overall performance versus data size. Arrival rate $=0.5$ no./s, deadline range $=[100,500]$.

when deadlines increase. The implication of this result is that loose deadlines lead to relatively low energy consumption. As such, the PARM algorithm significantly outperforms all the other alternatives in terms of overall performance. 
TABLE II

DESCRIPTIONS OF REAL COMMUNICATION-INTENSIVE APPLICATIONS

\begin{tabular}{|c|c|c|}
\hline Application & Average Message Size & Description \\
\hline Render & $512000 \mathrm{~B}$ & $\begin{array}{l}\text { The Render application integrates a } 60006000 \text { pixel } 24 \text {-bit color image } \\
\text { with 16-bits of elevation data per pixel to obtain } 480640 \text { pixel } 24 \text {-bit output images. }\end{array}$ \\
\hline Molecular & $45568 \mathrm{~B}$ & $\begin{array}{l}\text { The Molecular application is used to compute properties for liquids and polymers and } \\
\text { to calculate conformational properties of organic molecules. }\end{array}$ \\
\hline Semi & $8192 B$ & $\begin{array}{l}\text { The Semi application is a } 3 \text {-D semiconductor device simulator } \\
\text { that utilizes a parallelized } 3-\mathrm{D} \text { Poisson solver. }\end{array}$ \\
\hline React & 11264B & $\begin{array}{l}\text { The React application is used to predict the behavior of chemical } \\
\text { reactions based on first principles. }\end{array}$ \\
\hline Vortex & $1245 \mathrm{~B}$ & The Vortex application models the evolution of vortices in a 2-dimensional fluid. \\
\hline QCD & $72 B$ & $\begin{array}{l}\text { The Quantum chromodynamics application uses simulations to study how } \\
\text { quarks and gluons interact to form particles such as protons and neutrons. }\end{array}$ \\
\hline Exflow & 2248B & The Exflow application makes use of adaptive grids to simulate 3-D fluid flow around a fixed body. \\
\hline Climate & $505 \mathrm{~B}$ & $\begin{array}{l}\text { The Climate application performs long range studies of climate change using } \\
\text { a general circulation model of the earth's atmosphere. }\end{array}$ \\
\hline
\end{tabular}

\section{Effect of Data Size}

In this experiment, the impact of data size is evaluated on the five message-scheduling algorithms. The message-arrival rate is set to $0.5 \mathrm{no} . / \mathrm{s}$, and the deadlines are randomly generated in the range from 100 to $500 \mathrm{~s}$.

The empirical results are shown in Figs. 7 and 8. The first observation made from Fig. 7 is that when the data size increases, the missed rates of the three algorithms increase accordingly. This result can be explained by the fact that the increasing data size leads to high workloads, which in turn tends to reject more real-time messages.

Fig. 8 shows that PARM can substantially improve the overall performance over the four competitors. The performance improvement becomes more pronounced with increasing values of the data size. The implication of this result is that PARM is capable of noticeably improving the overall performance of real-time wireless networks where the average data size of messages is large.

The following section is going to present experiments based on real-world I/O-intensive applications. Although there are real-world applications that can be used for the simulations, it is still necessary to present the experimental results for synthetic data. The reason is that, synthetic data can be generated based on many simulation parameters. For example, synthetic data can be generated based on different deadlines, data sizes, or arrival rates, which can demonstrate the impact that these features have on the algorithm and prove the correctness of the algorithm. However, it is still necessary to validate PARM using real-world application data even though the results from synthetic data are promising.

\section{E. Real I/O-Intensive Applications}

Note that the experimental results presented in Section VI-B-D are generated from synthetic real-time messages. To validate the results of the synthetic workload, eight real-world communication-intensive applications are used in this group of experiments [9] as benchmarks to further evaluate the performance of PARM algorithm in comparison with the alternative approaches. Table II provides the descriptions of the eight applications, which have different computation, communication, and disk I/O patterns.

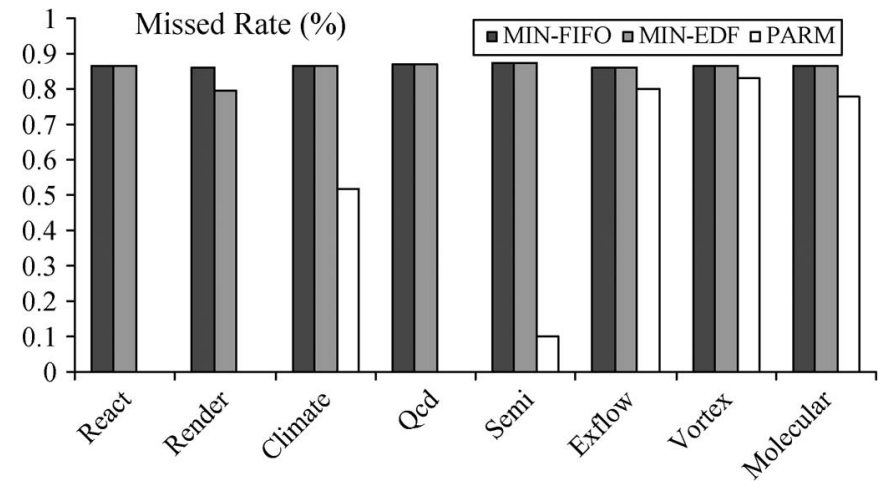

Fig. 9. Missed rates of the eight communication-intensive applications.

Fig. 9 shows the missed-rate values of the eight real-world applications. First, all the communication-intensive benchmarks benefit from the proposed PARM algorithm with respect to missed rate. Compared with the MIN_FIFO and MIN_EDF algorithms, PARM can significantly reduce the missed rate by up to $100 \%$ with an average of $56.10 \%$. Interestingly, the React, Render, and QCD applications take the best advantage of PARM by virtue of their low arrival rates. The performance improvements gained by the PARM algorithm become obvious for the real-world applications because PARM is capable of judiciously increasing the transmission rate under high workloads in order to guarantee timing constraints.

Second, MIN_FIFO and MIN_EDF yield approximately identical performance in missed rate for all the applications except for Render and React because of the low arrival rates of these two applications. This result indicates that the performance discrepancy between MIN_FIFO and MIN_EDF becomes less significant when the message arrival rate is higher than 0.23 no./ms. Third, compared with the other five benchmarks, the Exflow, Vortex, and Molecular applications under the PARM scheme exhibit high missed rates. Although the message data sizes of these three applications are relatively small, the arrival rates of these benchmarks are very high. This new finding suggests that, for all the tested applications, the performance in missed rate largely depends on arrival rate rather than message data size. Fig. 10 shows the mean power consumption of the real applications running in a wireless networks using the three evaluated schemes. It is observed from Fig. 10 that, with respect to power consumption, the PARM approach works 


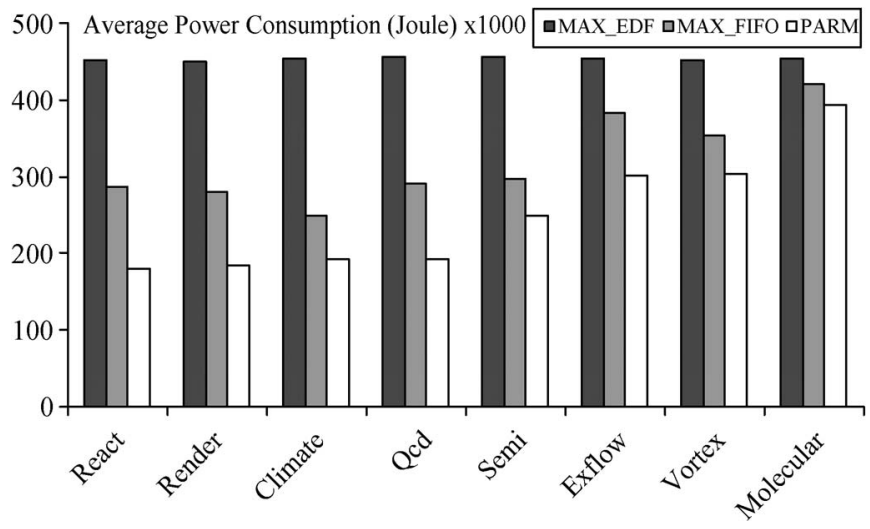

Fig. 10. Average power consumption of the eight applications.

quite well for all the communication-intensive applications. For example, compared with the two baseline algorithms, the PARM scheme significantly reduces energy dissipation by up to $60.0 \%$ (with an average of $34.1 \%$ ) over the three existing policies. PARM can achieve impressive performance gains in power consumption because PARM endeavors to save energy by decreasing transmission rates provided that the deadlines of real-time messages are met.

The energy-dissipation reduction varies from application to application. For example, the reduction is more pronounced for React, Render, Climate, QCD, and Semi than for Exflow, Vortex, and Molecular. These experimental results show that potential performance improvement gradually diminishes with increasing workloads, indicating that PARM can substantially reduce power consumption for applications with modest network traffic.

\section{CONCLUSION}

This paper has addressed the issue of scheduling real-time messages of multimedia applications in wireless networks subject to timing and power constraints. A PARM scheduling heuristic is developed to generate optimal schedules for messages with deadlines and power constraints. The PARM algorithm aims at minimizing energy consumption and the probability of missing deadlines for real-time messages transmitted in wireless networks. In particular, the proposed PARM scheme is energy-efficient by virtue of power-aware message scheduling for real-time messages. Before the presentation of PARM, a power-consumption model is also proposed to measure energy consumption quantitatively based on messagetransmission rates. The model is used by the PARM scheme to calculate energy consumed by a real-time message transmitted at a particular rate. To quantitatively evaluate the effectiveness and practicality of the proposed PARM scheme, extensive experiments are conducted using synthetic traces. Experimental results based on a wide variety of synthetic workloads and eight real-world applications show that PARM significantly improves the performance in terms of missed rate, energy consumption, and overall performance over four baseline schemes. For example, the overall performance improvement of PARM is up to $61 \%$ over the four baseline algorithms.
Future studies in this research can be performed in the following directions: first, extend PARM power-aware scheduling algorithm to deal with storage systems and second, modify PARM message-scheduling scheme to handle a large-scale wireless network.

\section{REFERENCES}

[1] T. F. Abdelzaher and K. G. Shin, "Combined task and message scheduling in distributed real-time systems," IEEE Trans. Parallel Distrib. Syst., vol. 10, no. 11, pp. 1179-1191, Nov. 1999.

[2] K. Akkaya and M. Younis, "An energy-aware QoS routing protocol for wireless sensor networks," in Proc. 23rd Int. Conf. Distrib. Comput. Syst. Workshops, May 2003, pp. 710-715.

[3] T. P. Baker, "Multiprocessor EDF and deadline monotonic schedulability analysis," in Proc. 24th IEEE Int. RTSS, 2003, pp. 120-129.

[4] T. P. Baker, "An analysis of EDF schedulability on a multiprocessor," IEEE Trans. Parallel Distrib. Syst., vol. 16, no. 8, pp. 760-768, Aug. 2005.

[5] O. Beaumont, L. Marchal, V. Rehn, and Y. Robert, "FIFO scheduling of divisible loads with return messages under the one-port model," in Proc. 20th IPDPS, Apr. 2006, p. 14.

[6] C.-Y. Chang, J.-P. Sheu, Y.-C. Chen, and S.-W. Chang, "An obstacle-free and power-efficient deployment algorithm for wireless sensor networks," IEEE Trans. Syst., Man, Cybern. A, Syst., Humans, vol. 39, no. 4, pp. 795806, Jul. 2009.

[7] S.-C. Cheng and Y.-M. Huang, "Dynamic real-time scheduling for multi-processor tasks using genetic algorithm," in Proc. 28th Annu. Int. COMPSAC, Sep. 2004, vol. 1, pp. 154-160.

[8] C.-C. Chou, C.-S. Chang, D.-S. Lee, and J. Cheng, "A necessary and sufficient condition for the construction of 2-to-1 optical FIFO multiplexers by a single crossbar switch and fiber delay lines," IEEE Trans. Inf. Theory, vol. 52, no. 10, pp. 4519-4531, Oct. 2006.

[9] R. Cypher, A. Ho, S. Konstantinidou, and P. Messina, "Architectural requirements of parallel scientific applications with explicit communication," SIGARCH Comput. Archit. News, vol. 21, no. 2, pp. 2-13, 1993.

[10] A. El Gamal, C. Nair, B. Prabhakar, E. Uysal-Biyikoglu, and S. Zahedi, "Energy-efficient scheduling of packet transmissions over wireless networks," in Proc. IEEE 21st Annu. Joint Conf. IEEE Comput. Commun. Soc., INFOCOM, 2002, vol. 3, pp. 1773-1782.

[11] J. Gomez, A. Campbell, M. Naghshineh, and C. Bisdikian, "Paro: Supporting dynamic power controlled routing in wireless ad hoc networks," J. ACM Wirel. Netw., vol. 9, pp. 443-460, Jun. 2001.

[12] S. R. Gulliver and G. Ghinea, "A perceptual comparison of empirical and predictive region-of-interest video," IEEE Trans. Syst., Man, Cybern. A, Syst., Humans, vol. 39, no. 4, pp. 744-753, Jul. 2009.

[13] P. J. M. Havinga and G. J. M. Smit, "Energy-efficient wireless networking for multimedia applications," Wirel. Commun. Mobile Comput., vol. 1, no. 2, pp. 165-184, Apr--Jun. 2001.

[14] P. J. M. Havinga, G. J. M. Smit, and M. Bos, "Energy-efficient adaptive wireless network design," in Proc. 5th IEEE ISCC, 2000, pp. 502-507.

[15] J.-L. Huang, "AIDOA: An adaptive and energy-conserving indexing method for on-demand data broadcasting systems," IEEE Trans. Syst., Man, Cybern. A, Syst., Humans, vol. 38, no. 2, pp. 331-345, Mar. 2008.

[16] C. E. Jones, K. M. Sivalingam, P. Agrawal, and J. C. Chen, "A survey of energy efficient network protocols for wireless networks," Wirel. Netw., vol. 7, no. 4, pp. 343-358, Aug. 2001.

[17] M. A. Khojastepour and A. Sabharwal, "Delay-constrained scheduling: Power efficiency, filter design, and bounds," in Proc. 23rd Annu. Joint Conf. IEEE Comput. Commun. Soc., INFOCOM, Mar. 2004, vol. 3, pp. 1938-1949.

[18] G. Lipari and G. Buttazzo, "Scheduling real-time multi-task applications in an open system," in Proc. 11 th Euromicro Conf. Real-Time Syst., 1999, pp. 234-241.

[19] C. L. Liu and J. W. Layland, "Scheduling algorithms for multiprogramming in a hard-real-time environment," J. ACM, vol. 20, no. 1, pp. 46-61, Jan. 1973.

[20] W. Luo, X. Qin, X.-C. Tan, K. Qin, and A. Manzanares, "Exploiting redundancies to enhance schedulability in fault-tolerant and real-time distributed systems," IEEE Trans. Syst., Man, Cybern. A, Syst., Humans, vol. 39, no. 3, pp. 626-639, May 2009.

[21] M. Nijim, X. Qin, and T. Xie, "Adaptive quality of security control in networked parallel disk systems," in Proc. 15th ICCCN, Oct. 2006, pp. 455-460. 
[22] M. Nijim, X. Qin, and T. Xie, "Modeling and improving security of a local disk system for write-intensive workloads," ACM Trans. Storage, vol. 2, no. 4, pp. 400-423, Nov. 2006.

[23] B. Prabhakar, E. U. Biyikoglu, and A. El Gamal, "Energy-efficient transmission over a wireless link via lazy packet scheduling," in Proc. IEEE 20th Annu. Joint Conf. IEEE Comput. Commun. Soc., INFOCOM, 2001, vol. 1, pp. 386-394.

[24] X. Qin and H. Jiang, "Dynamic, reliability-driven scheduling of parallel real-time jobs in heterogeneous systems," in Proc. Int. Conf. Parallel Process., Sep. 2001, pp. 113-122.

[25] X. Qin and H. Jiang, "A dynamic and reliability-driven scheduling algorithm for parallel real-time jobs executing on heterogeneous clusters," J. Parallel Distrib. Comput., vol. 65, no. 8, pp. 885-900, Aug. 2005.

[26] S. Shenker, D. D. Clark, and L. Zhang, "Supporting real-time applications in an integrated services packet network: Architecture and mechanism," in Proc. ACM Conf. SigComm, 1992, pp. 14-26.

[27] S. Singh and C. S. Raghavendra, "PAMAS-Power aware multi-access protocol with signalling for ad hoc networks," SIGCOMM Comput. Commun. Rev., vol. 28, no. 3, pp. 5-26, 1998.

[28] J. A. Stine and G. De Veciana, "Improving energy efficiency of centrally controlled wireless data networks," Wirel. Netw., vol. 8, no. 6, pp. 681-700, Nov. 2002.

[29] M. E. Thomadakis and J.-C. Liu, "On the efficient scheduling of nonperiodic tasks in hard real-time systems," in Proc. 20th IEEE RTSS, 1999, pp. 148-151.

[30] D. W. Tjondronegoro and Y.-P. P. Chen, "Knowledge-discounted event detection in sports video," IEEE Trans. Syst., Man, Cybern. A, Syst., Humans, vol. 40, no. 5, pp. 1009-1024, Sep. 2010.

[31] E. Uysal-Biyikoglu, B. Prabhakar, and A. El Gamal, "Energy-efficient packet transmission over a wireless link," IEEE/ACM Trans. Netw., vol. 10, no. 4, pp. 487-499, Aug. 2002.

[32] X. Wang and W. Wang, "Energy-aware call admission control scheme in wireless cellular networks," in Proc. IEEE GLOBECOM, Nov. 29Dec. 3, 2004, vol. 3, pp. 1570-1574.

[33] T. Xie and X. Qin, "Enhancing security of real-time applications on grids through dynamic scheduling," in Proc. JSSPP, 2005, pp. 219-237.

[34] T. Xie and X. Qin, "A new allocation scheme for parallel applications with deadline and security constraints on clusters," in Proc. IEEE Int. Cluster Comput., Sep. 2005, pp. 1-10.

[35] T. Xie and X. Qin, "Scheduling security-critical real-time applications on clusters," IEEE Trans. Comput., vol. 55, no. 7, pp. 864-879, Jul. 2006.

[36] T. Xie and X. Qin, "Improving security for periodic tasks in embedded systems through scheduling," ACM Trans. Embedded Comput. Syst., vol. 6, no. 3, p. 20, Jul. 2007.

[37] G. N. Yannakakis, M. Maragoudakis, and J. Hallam, "Preference learning for cognitive modeling: A case study on entertainment preferences," IEEE Trans. Syst., Man, Cybern. A, Syst., Humans, vol. 39, no. 6, pp. 1165-1175, Nov. 2009.

[38] H. Zhu and G. Cao, "A power-aware and QoS-aware service model on wireless networks," in Proc. 23rd Annu. Joint Conf. IEEE Comput. Commun. Soc., INFOCOM, Mar. 2004, vol. 2, pp. 1393-1403.

[39] H. Zhu and G. Cao, "On supporting power-efficient streaming applications in wireless environments," IEEE Trans. Mobile Comput., vol. 4, no. 4, pp. 391-403, Jul./Aug. 2005.

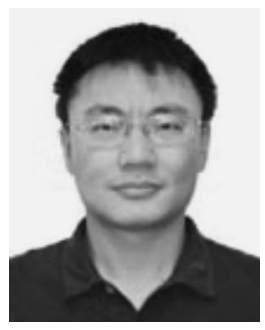

Xiaojun Ruan (S'08-A'10) received the B.S. degree in computer science and technology from Shandong University, Jinan, China, in 2005. He is currently working toward the Ph.D. degree in the Department of Computer Science and Software Engineering, Auburn University, Auburn, AL.

His research interests focus on parallel and distributed systems, high-performance parallel cluster computing, storage systems, energy-efficient cluster systems, solid-state disks, real-time systems, and network security.

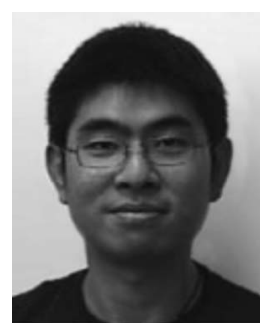

Shu Yin (S'09) received the B.S. degree in communication engineering and the M.S. degree in signal and information processing from the Wuhan University of Technology, Wuhan, China, in 2006 and 2008 , respectively. He is currently working toward the Ph.D. degree in the Department of Computer Science and Software Engineering, Auburn University, Auburn, AL.

His research interests include storage systems, reliability modeling, fault tolerance, energy-efficient computing, and wireless communications.

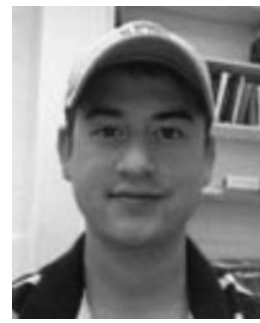

Adam Manzanares (S'08-M'10) received the B.S. degree in computer science from the New Mexico Institute of Mining and Technology, Socorro, and the Ph.D. degree from the Department of Computer Science and Software Engineering, Auburn University, Auburn, AL, in 2006 and 2010, respectively.

During the summers of 2002-2007, he was a Student Intern with the Los Alamos National Laboratory, Los Alamos, NM, where he is currently a Postdoctoral Fellow. His research interests include energy-efficient computing, modeling and simulation, and high-performance computing.

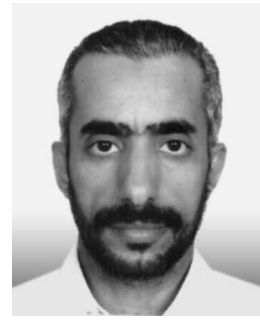

Mohammed Alghamdi received the B.S. degree in computer science from King Saud University, Riyadh, Saudi Arabia, in 1997, the M.S. degree in software engineering from Colorado Technical University, Denver, the M.S. degree in information technology management from Colorado Technical University, in 2003, and the Ph.D. degree in computer science from the New Mexico Institute of Mining and Technology, Socorro. He is currently an Assistant Professor in the Department of Computer Science, Al-Baha University, Al-Baha, Saudi Arabia.

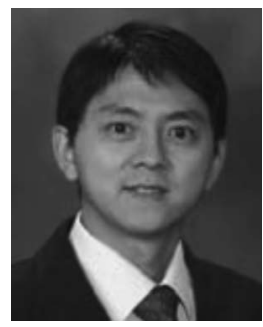

Xiao Qin (S'00-M'04-SM'09) received the B.S and M.S. degrees in computer science from the Huazhong University of Science and Technology, Wuhan, China, and the Ph.D. degree in computer science from the University of Nebraska-Lincoln, Lincoln, in 1992, 1999, and 2004, respectively.

For three years, he was an Assistant Professor with the New Mexico Institute of Mining and Technology, Socorro. Currently, he is an Associate Professor with the Department of Computer Science and Software Engineering, Auburn University, Auburn, AL. His research interests include parallel and distributed systems, storage systems, fault tolerance, real-time systems, and performance evaluation. His research is supported by the U.S. National Science Foundation, Auburn University, and Intel Corporation.

Dr. Qin was a recipient of the U.S. National Science Foundation (NSF) Computing Processes and Artifacts Award and the NSF Computer System Research Award in 2007 and the NSF CAREER Award in 2009. He was a Subject Area Editor of the IEEE Distributed System Online in 2000-2001. He has been on the program committees of various international conferences, including IEEE Cluster, IEEE International Performance, Computing, and Communications Conference, and International Conference on Parallel Processing. 